\title{
Moderne Kardiologie anstelle von Innerer Medizin - ist das die richtige Strategie für eine prosperierende Zukunft der Medizin?
}

Barbara C. Biedermann

Korrespondenz:

PD Dr. med. Barbara C. Biedermann Medizinische Universitätsklinik Kantonsspital Bruderholz CH-4101 Bruderholz
Was wie eine dogmatische Lehrschrift der Kardiologie und der modernen Medizin geschrieben und veröffentlicht wurde [1], darf nicht unbeantwortet bleiben. Dass einer der führenden Repräsentanten und akademischen Lehrer der Schweiz eines der wichtigsten Spezialgebiete der Inneren Medizin, nämlich der Kardiologie, einfach Glaubenssätze formuliert (Beispiel: «Im tertiären Bereich funktioniert die Kardiologie problemlos als losgelöste Einheit»), fordert Widerstand heraus.

Bezeichnenderweise untermauert der Autor seine Aussagen mit keinem einzigen Literaturhinweis. Wenn man Unwissende oder Zweifelnde in Glaubensfragen überzeugen möchte (und das scheint eines der Anliegen dieses Artikels), empfiehlt sich heutzutage breitabgestützte Argumentation. Das haben selbst die Päpste der römisch-katholischen Kirche, einer Institution, der Kritiker oft Dogmatik zum Vorwurf machen, längst gelernt [2]. Doch die Medizin ist nicht eine Religion und Dogmatik hat in der modernen Medizin keinen Platz.

Ohne die für betroffene Patienten unbestrittenermassen grossartigen Leistungen der invasiven Kardiologie schmälern zu wollen, vermisse ich an dieser Gesamtschau [1] modernes, differenziertes Krankheitsverständnis: der Arteriosklerose, der arteriellen Hypertonie, des Vorhofflimmerns, der Herzinsuffizienz usw. Lassen Sie mich drei Gegenthesen zu diesem Artikel aufstellen und sie mit Argumenten untermauern.

\section{Die Innere Medizin ist mehr als die Summe ihrer Spezialitäten}

Die Arteriosklerose ist heute zwar die wichtigste Ursache eines Herzinfarktes. Doch obschon sich die Arteriosklerose im koronaren Gefässbett für den betroffenen Patienten verheerend auswirkt, ist sie oft eine panarterielle Erkrankung [3]. Die perkutane Angioplastie oder die Bypass-Operation kann deshalb am «Ort der Not» zwar wirksam Abhilfe schaffen, wenn jedoch nicht panarteriell, d.h. systemisch behandelt wird, folgen mit grosser Wahrscheinlichkeit weitere, zum Teil schleichende Organmanifestationen wie vaskuläre Demenz, Niereninsuffizienz, sekundäre arterielle Hypertonie, um nur die wichtigsten zu nennen. Die Entwicklung gutwirksamer Medikamente zur systemischen Behandlung der Arteriosklerose kann nur mit dem präzisen, molekularen Verständnis der Grundkrankheit gelingen. Einem Ziel, dem die Medizin heute greifbar nahe ist. Solche Medikamente sollen die Arteriosklerose nicht nur am Fortschreiten hindern, sondern sie in Remission bringen.

\section{Fazit}

Die Arteriosklerose als chronische, panarterielle und organübergreifende Erkrankung wird in nicht allzu ferner Zukunft am besten wieder vom Internisten behandelt werden.

\section{2. Ökonomisches Gewicht ist nicht mit inhaltlicher Bedeutung gleichzusetzen}

Die Porphyrie ist eine seltene, hereditäre Krankheit, wirtschaftlich auf den ersten Blick völlig uninteressant, eine sogenannte «orphan disease». Die Porphyrie verläuft schubweise, und Schübe können durch bestimmte Medikamente ausgelöst werden. Die Aufklärung der molekularen Wirkweise schubauslösender Medikamente hat nicht nur die Entwicklung einer simplen Behandlung (nämlich Hämarginat) des akuten PorphyrieSchubs ermöglicht [4], sondern sie hat auch zur Beschreibung und zum Verständnis des Cytochrom-P450-Systems der Leber beigetragen. Das Cytochrom-P450-System baut viele Medikamente und Fremdsubstanzen im Körper ab. Die individuelle Zusammensetzung und Ausprägung dieses Systems bestimmt oft Wirkung und Nebenwirkung von Medikamenten [5]. Dass gewisse Betablocker bei bestimmten Patienten schlechter verträglich sind als andere, ist auf ihren Metabolismus durch ein bestimmtes Cytochrom P450 zurückzuführen.

\section{Fazit}

Grundlagenforschung über eine seltene Krankheit hat zum Verständnis des variablen Neben- 
wirkungsprofils einer der am häufigsten verschriebenen Medikamentenklassen geführt und letztlich den wirtschaftlichen Misserfolg eines Medikaments erklärt.

\section{3. (Spitzen-)Technologisierung ersetzt nicht das präzise Krankheitsverständnis (sondern wird gelegentlich durch letzteres ersetzt)}

Bis vor wenigen Jahren war die allogene Knochenmarkstransplantation die einzige Heilungsmöglichkeit für Patienten mit chronisch myeloischer Leukämie. Die Erkenntnis, dass eine bestimmte Chromosomentranslokation die chronisch myeloische Leukämie in den meisten Fällen auslöst und dass diese Chromosomentranslokation zu einer verstärkten Aktivierung einer sogenannten Tyrosinkinase führt, die die betroffene myelopoietische Stammzelle zur Proliferation anregt, bildete die Grundlage für die Entwicklung eines erstaunlich wirksamen und gutverträglichen Medikamentes zur Behandlung dieser Leukämie [6].

\section{Fazit}

Die Entwicklung eines gezielt, quasi am molekularen Ursprung einer Krankheit wirksamen Medikaments kann über Nacht ein hochtechnologisiertes, spitzenmedizinisches Behandlungsverfahren als Erstlinientherapie ersetzen.

Im letzten Satz des Artikels von Prof. Dr. med. B. Meier wird ein Instrument erwähnt, mit dem mittelalterliche Inquisitoren Ketzer endgültig zum Schweigen brachten: der Scheiterhaufen. Der Autor hat recht: Fortschritt und Aufklärung lassen sich mit solchen Mitteln nicht aufhalten - das lehrt uns die Geschichte. Gutes und Richtiges setzt sich langfristig durch. Das galt in den 70er Jahren des vorigen Jahrhunderts für die perkutane Angioplastie genauso wie heute für die molekulare Diagnose und Therapie der Arteriosklerose. Deshalb sollen die heutigen Medizinstudenten (sie sind die Anwälte der Patienten von morgen!) mutig ihre Lehrer einer modernen Medizin fragen, welches die präzisen Krankheitsursachen sind. Erst das genaue Krankheitsverständnis bildet die Voraussetzung für eine korrekte Diagnose und wirksame, nachhaltige
Therapie. Am Tag, an dem Patienten mit Arteriosklerose mit Hilfe molekular wirkender Medikamente in Remission kommen können, bevor sie einen Herzinfarkt erleiden, wird die Mehrheit der interventionellen Kardiologen das gleiche Schicksal ereilen wie seinerzeit die Ulkus-Chirurgen, als die effizienten Protonenpumpenhemmer Marktreife erlangten [7] und als Helicobacter pylori als weitere Ulkus-Ursache erkannt wurde [8].

Etwas sollten wir alle, die wir Medizin lehren und durch Forschung weiterentwickeln, von der Inneren Medizin lernen: Sie bezieht ihre Stärke nicht durch Ausgrenzen und Fragmentieren, sondern durch Integration und Synthese. So jedenfalls habe ich dieses Fach von meinen verehrten Lehrern gelernt und ich wünsche meinen jungen Kolleginnen und Kollegen ebensolche Vorbilder. Ungeachtet, ob sie später Hausärzte, Chirurgen, Internisten, Gynäkologen oder Radiologen werden.

\section{Literatur}

1 Meier B. Moderne Kardiologie - eine Gesamtschau. Schweiz Ärztezeitung 2006;87(15/16):669-73.

2 Benedikt XVI. Deus caritas est. Augsburg: St. Ulrich Verlag; 2005.

3 Fleiner M, Kummer M, Mirlacher M, et al. Arterial neovascularization and inflammation in vulnerable patients: early and late signs of symptomatic atherosclerosis. Circulation 2004;110(18):2843-50.

4 Tokola O, Tenhunen R, Volin L, Mustajoki P. Pharmacokinetics of intravenously administered haem arginate. Br J Clin Pharmacol 1986;22(3):331-5.

5 Meyer UA, Skoda RC, Zanger UM. The genetic polymorphism of debrisoquine/sparteine metabolism-molecular mechanisms. Pharmacol Ther 1990;46(2):297-308

6 Druker BJ, Sawyers CL, Kantarjian H, et al. Activity of a specific inhibitor of the BCR-ABL tyrosine kinase in the blast crisis of chronic myeloid leukemia and acute lymphoblastic leukemia with the Philadelphia chromosome. N Engl J Med 2001;344(14):1038-42.

7 Gustavsson S, Adami HO, Loof L, Nyberg A, Nyren O. Rapid healing of duodenal ulcers with omeprazole: double-blind dose-comparative trial. Lancet 1983;2(8342):124-5.

8 Marshall BJ, Warren JR. Unidentified curved bacilli in the stomach of patients with gastritis and peptic ulceration. Lancet 1984;1(8390):1311-5. 